\title{
Improving Benefit-harm Assessment of Therapies from the Patient Perspective: OMERACT Premeeting Toward Consensus on Core Sets for Randomized Controlled Trials
}

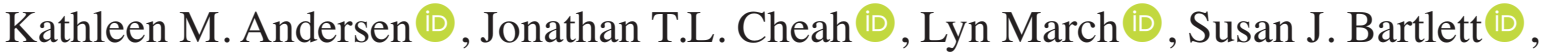 \\ Dorcas Beaton (1D), Clifton O. Bingham III ${ }^{\circledR}$, Peter M. Brooks (1), Robin Christensen (1), \\ Philip G. Conaghan (1), Maria-Antonietta D’Agostino (D), Maarten de Wit (D), \\ Amylou C. Dueck (D), Susan M. Goodman (1), Shawna Grosskleg (1D, Catherine L. Hill (1), \\ Martin Howell (D), Sarah L. Mackie (D), Bethan Richards (D), Beverly Shea (D),

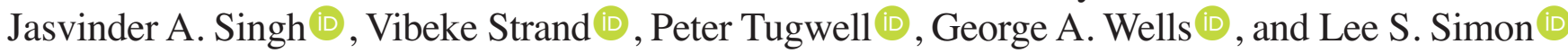

ABSTRACT. Objective. Outcome Measures in Rheumatology (OMERACT) convened a premeeting in 2018 to bring together patients, regulators, researchers, clinicians, and consumers to build upon previous OMERACT drug safety work, with patients fully engaged throughout all phases.

Methods. Day 1 included a brief introduction to the history of OMERACT and methodology, and an overview of current efforts within and outside OMERACT to identify patient-reported medication safety concerns. On Day 2, two working groups presented results; after each, breakout groups were assembled to discuss findings.

Results. Five themes pertaining to drug safety measurement emerged.

Conclusion. Current approaches have failed to include data from the patient's perspective. A better understanding of how individuals with rheumatic diseases view potential benefits and harms of therapies is essential. (First Release February 15 2019; J Rheumatol 2019;46:1053-8; doi:10.3899/ jrheum.181123)

Key Indexing Terms:

OMERACT PATIENT SATISFACTION

DISEASE-MODIFYING ANTIRHEUMATIC DRUGS

CLINICAL TRIALS

RISK ASSESSMENT

From the Department of Family Medicine, and Department of Medicine, McGill University, Montreal, Quebec; Institute for Work \& Health, Toronto; OMERACT; Ottawa Hospital Research Institute, Ottawa Hospital; Division of Rheumatology, Department of Medicine, and School of Epidemiology and Public Health, Faculty of Medicine, University of Ottawa; Clinical Epidemiology Program, Ottawa Hospital Research Institute, Ottawa Cardiovascular Research Methods Centre, University of Ottawa Heart Institute, Ottawa, Ontario, Canada; Musculoskeletal Statistics Unit, The Parker Institute, Bispebjerg and Frederiksberg Hospital, Copenhagen; Department of Rheumatology, Odense University Hospital, Odense, Denmark; Division of Rheumatology, Hospital for Special Surgery; Department of Medicine, Weill Cornell Medical School, New York, New York; Division of Rheumatology, Department of Medicine Johns Hopkins University, Baltimore, Maryland; Division of Biostatistics, Department of Health Sciences Research, Mayo Clinic, Scottsdale, Arizona; Medicine Service, VA Medical Center; Department of Medicine, School of Medicine, University of Alabama at Birmingham; Division of Epidemiology, School of Public Health, University of Alabama at Birmingham, Birmingham, Alabama; Division of Immunology/

Rheumatology, Stanford University, Palo Alto, California; SDG LLC, Cambridge, Massachusetts, USA; Sydney Medical School, and Sydney School of Public Health, University of Sydney; Centre for Kidney Research, The Children's Hospital at Westmead, Sydney; Institute of Bone and Joint Research, Kolling Institute, Northern Sydney Local Health District; Department of Rheumatology, Royal North Shore Hospital, Reserve Road, St Leonards; Centre for Health Policy, School of Population and Global Health, The University of Melbourne, Melbourne; Discipline of Medicine, University of Adelaide; Rheumatology Unit, The Queen Elizabeth Hospital, Woodville; Department of Rheumatology, Royal
Prince Alfred Hospital, Camperdown, Australia; Leeds Institute of Rheumatic and Musculoskeletal Medicine, University of Leeds; UK National Institute for Health Research (NIHR) Leeds Biomedical Research Centre, Leeds Teaching Hospitals National Health Service (NHS) Trust; NIHR-Leeds Musculoskeletal Biomedical Research Unit, University of Leeds, Leeds, UK; Hôpital Ambroise Paré, Rheumatology Department, Boulogne-Billancourt; INSERM U1173, Laboratoire d'Excellence INFLAMEX, UFR Simone Veil, Versailles-Saint-Quentin University, SaintQuentin en Yvelines, France; Amsterdam University Medical Centre, Department of Medical Humanities, Amsterdam Public Health, Amsterdam, the Netherlands.

The Parker Institute, Bispebjerg and Frederiksberg Hospital (RC) is supported by a core grant from the Oak Foundation (OCAY-13-309). PGC is supported in part by the NIHR Leeds Biomedical Research Centre. The views expressed are those of the authors and not necessarily those of the NHS, the NIHR or the Department of Health.

K.M. Andersen, MSc, Department of Family Medicine, McGill University, and Musculoskeletal Statistics Unit, The Parker Institute, Bispebjerg and Frederiksberg Hospital; J.T. Cheah, MBBS, Rheumatology Fellow, Division of Rheumatology, Hospital for Special Surgery, and Fellow in Medicine, Department of Medicine, Weill Cornell Medicine; L. March, MBBS, MSc, PhD, FRACP, FAFPHM, Sydney Medical School, University of Sydney, and Institute of Bone and Joint Research, Kolling Institute, Northern Sydney Local Health District, and Department of Rheumatology, Royal North Shore Hospital; S.J. Bartlett, PhD, Professor, Department of Medicine, McGill University, and Adjunct Professor, Department of Medicine, Johns Hopkins University; D. Beaton, BscOT, PhD, Senior Scientist, Institute for Work \& Health; C.O. Bingham III, MD, Division of Rheumatology, Department of Medicine, Johns Hopkins University;

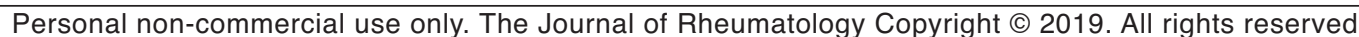


Outcome Measures in Rheumatology (OMERACT) is an international initiative aimed at improving outcome measurement across rheumatologic conditions. Immediately prior to the OMERACT 2018 biannual event, a special premeeting was convened entitled "Improving Risk-Benefit Assessment of Drugs, with an Emphasis on Patients and their Perspectives" on May 13-14, 2018, in Terrigal, New South Wales, Australia. The meeting was designed to bring together people reflecting a variety of perspectives to discuss current policies and approaches in patient-focused drug development, and review ongoing work by OMERACT and other initiatives in this area ${ }^{1,2,3,4}$. Notably, because this meeting included representatives from multiple regulatory and pharmaceutical industries from around the world, it offered a unique opportunity to hear global perspectives on the growing importance of patient engagement in regulatory affairs.

While OMERACT has a legacy of work in this area, notable research over the past 2 years represented a fresh look at drug safety. Consistent with OMERACT principles, in this work patients were fully engaged as patient research partners

P.M. Brooks, MD, Centre for Health Policy, School of Population and Global Health, The University of Melbourne; R. Christensen, MSc, PhD, Professor of Biostatistics and Clinical Epidemiology, Musculoskeletal Statistics Unit, The Parker Institute, Bispebjerg and Frederiksberg Hospital, and Department of Rheumatology, Odense University Hospital; P.G. Conaghan, MBBS, PhD, Director, Leeds Institute of Rheumatic and Musculoskeletal Medicine, University of Leeds, and Deputy Director, NIHR Leeds Biomedical Research Centre, Leeds Teaching Hospitals NHS Trust; M.A. D'Agostino, MD, PhD, APHP, Hôpital Ambroise Paré, Rheumatology Department, and INSERM U1173, Laboratoire d'Excellence INFLAMEX, UFR Simone Veil; M. de Wit, PhD, Amsterdam University Medical Centre, Department of Medical Humanities, Amsterdam Public Health; A. Dueck, PhD, Division of Biostatistics, Department of Health Sciences Research, Mayo Clinic; S.M. Goodman, MD, Professor of Clinical Medicine, Division of Rheumatology, Hospital for Special Surgery, and Professor of Clinical Medicine, Department of Medicine, Weill Cornell Medical School; S. Grosskleg, OMERACT, and University of Ottawa; C.L. Hill, MBBS, MSc, MD, FRACP, Professor, Discipline of Medicine, University of Adelaide \& Rheumatology Unit, The Queen Elizabeth Hospital; M. Howell, PhD, Sydney School of Public Health, The University of Sydney, and Centre for Kidney Research, The Children's Hospital at Westmead; S.L. Mackie, MB BCh, PhD, MRCP, Associate Clinical Professor, NIHR-Leeds Musculoskeletal Biomedical Research Unit, University of Leeds; B. Richards, MBBS (Hons), MClinEpi, MSportsMed, FRACP, Department of Rheumatology, Royal Prince Alfred Hospital; B. Shea, PhD, Clinical Investigator, Ottawa Hospital Research Institute, Ottawa Hospital, and Adjunct Professor, Public Health, Faculty of Medicine, University of Ottawa; J.A. Singh, MBBS, MPH, Medicine Service, VA Medical Center, and Department of Medicine, School of Medicine, University of Alabama at Birmingham, and Division of Epidemiology, School of Public Health, University of Alabama at Birmingham; V. Strand, MD, Biopharmaceutical Consultant, Portola Valley, California, USA; P. Tugwell, MD, MSc, Division of Rheumatology, Department of Medicine, and School of Epidemiology and Public Health, Faculty of Medicine, University of Ottawa, and Clinical Epidemiology Program, Ottawa Hospital Research Institute; G.A. Wells, MSc, PhD, School of Epidemiology and Public Health, University of Ottawa, and Cardiovascular Research Methods Centre, University of Ottawa Heart Institute; L.S. Simon, MD, SDG LLC.

Address correspondence to K.M. Andersen, Department of Family Medicine, McGill University, 5858 chemin de la Cote-des-Neiges, Montreal, Quebec H3S 1Z1, Canada.

E-mail: kathleen.andersen@mail.mcgill.ca

Accepted for publication December 11, 2018.
(PRP) throughout all phases of the work, from conceptualization through interpretation of results.

The specific aims of the meeting were to invite our PRP to (1) convene with many of those involved to review ongoing global efforts in patient-focused drug development; (2) identify opportunities for co-learning and development of patient-centered methods to assess potential harms in rheumatology, oncology, and nephrology clinical trials; and (3) develop this paper outlining key considerations for the development of core outcome sets and measures of patientvalued safety outcomes for use in randomized controlled trials (RCT).

\section{MATERIALS AND METHODS}

There were 42 participants (9 PRP, 2 rheumatology fellows, 26 clinician/researchers, and 5 regulators, payers, or industry scientists; some individuals contributed to multiple categories) and new and returning OMERACT members. A professional scribe created visual representations of the discussions on a white board throughout the meeting.

During the first day, a brief introduction to the history of OMERACT and current methodologies focusing on previous drug safety work was presented by OMERACT executive members along with a brief overview of the new OMERACT Filter 2.1 $1^{5,6}$ approaches to core set development (Figure 1). Current patient-centered efforts to assess benefits and harms were presented from PRP and regulatory representatives from the US Food and Drug Administration, the Canadian Agency for Drugs and Technologies in Health, the European Medicines Agency, the Ministry of Health New Zealand, and the Pharmaceutical Benefits Advisory Committee of Australia. Colleagues from nephrology ${ }^{7}$ and oncology ${ }^{8}$ presented new patient-reported outcomes (PRO) querying side effects and adverse events in their fields.

On the second day, 2 OMERACT working groups presented new results investigating patient attitudes and experiences with rheumatology therapies. The OMERACT Safety Group presented results from 6 focus groups of patients with inflammatory arthritis in Canada, the United States, and Australia regarding their experiences and considerations with diseasemodifying antirheumatic drugs. The OMERACT Glucocorticoid Impact Group summarized work completed over the past 2 years including 2 literature reviews, a survey, and patient interviews used to inform an ongoing Delphi to prioritize patient-valued outcomes regarding steroid use in rheumatology. Following each presentation, breakout groups of 8-10 people were assembled to discuss key findings, implications, and opportunities and to identify additional work needed. The full group was reconvened, and a representative from each group summarized the discussion and key messages for all attendees.

\section{RESULTS}

The initial presentations introduced attendees to OMERACT's longstanding commitment to fully engaging PRP as co-producers in the development and validation of outcome measures in rheumatology. Patient attendees then discussed the challenges that many of them had faced while understanding the relative benefits and harms of therapeutics, how discussions (or lack thereof) with providers influenced their perceptions of safety and effectiveness, and individual considerations regarding safety that reflected personal priorities and values. Consensus quickly emerged that clinicians and trialists who monitor safety in drug development often focus on different outcomes from those that patients value most. For example, patients taking methotrexate to control 




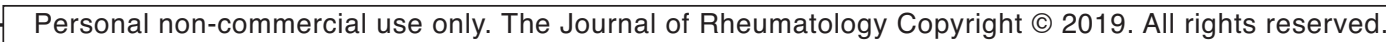


their disease often reported considerable effect of what are often termed "nuisance side effects" (mental fog, nausea, and gastrointestinal upset) on quality of life. In contrast, clinicians are primarily concerned with pathophysiologic manifestations such as hepatotoxicity when monitoring the effects of treatment.

Next, examples of patient-centered safety monitoring strategies in nephrology and oncology were presented. A representative from the Standardised Outcomes in Nephrology (SONG) Initiative briefly summarized ongoing work to identify patient-valued core domain sets and measures for use in nephrology trials across a range of diseases. Similar to rheumatology, the nephrology community views current reporting of harms in RCT as poorly defined, inadequate, unreliable, and failing to identify the range of patient experiences. Adapted from the OMERACT onion $^{5}$, SONG has a conceptual schema of a kidney that represents disease-specific mandatory and discretionary outcomes that the nephrology community recommends be measured in trials ${ }^{9}$.

While a PRO assessing potential harms is not yet available in rheumatology or nephrology, a measure has been developed and extensively validated in oncology ${ }^{10}$. The US National Cancer Institute's Patient-Reported Outcomes version of the Common Terminology Criteria for Adverse Events (PRO-CTCAE) consists of a bank of 124 patient-reported items describing 78 symptomatic adverse events such as dysphagia, nausea, and sensory neuropathy in the context of cancer treatment ${ }^{11,12}$. Importantly, PRO-CTCAE moves beyond binary presence or absence of symptoms, and asks about frequency, severity, and interference with daily activities (where applicable) of each symptom. This represents a major advancement in more fully characterizing patient experiences. There was consensus among attendees during discussion following the presentation that it is important to fully identify relevant aspects of symptoms when designing a rheumatology safety PRO. The PRO-CTCAE item bank also allows investigators to tailor symptom queries based on a specific molecule or drug class, and separates treatment-related effects from overall disease burden. Importantly, the PRO-CTCAE is typically used for weekly reporting for treatment that is delivered during a defined period, which could be applicable to weekly reporting in rheumatology trials but may not be feasible in rheumatology clinical practice, where treatment is generally over a longer time period.

We summarize here the overarching themes resulting from the small and large group discussions of the OMERACT Safety and Glucocorticoid Toxicity Groups, and proposed ways to proceed (Figure 2). First, there was consensus that patients, their families, and caregivers often have differing priorities and expectations of benefits and harms from their clinicians and trialists. Discrepancies between patients and clinicians on what matters most were echoed by results from
SONG, where patients once again reported higher concern about effects on everyday life (fatigue, negative emotions) while physicians reported greater concern about clinically defined medical events (cardiac arrest, heart attack, stroke, heart failure $)^{13}$. Further, in a cluster-randomized trial, oncologists who were provided with their patient's PRO-CTCAE scores were significantly more likely to report important symptomatic adverse events that patients also reported (pain, anxiety, fatigue, anorexia, dysphagia, depression) than did oncologists who did not receive their patient's PRO-CTCAE scores ${ }^{14}$. Thus, attendees agreed it is essential to consider many perspectives when identifying essential domains to include in core outcome sets.

Second, to identify the effect of safety events from the patient perspective, it is important to ask patients about the effect of medication-related symptoms on day-to-day life, because the cumulative effects over time appear to be a key driver of patient priorities. It may also be helpful for patients to ask their family and friends if they have noticed changes in their physical, emotional, and social function that are potentially treatment-related. Attendees acknowledged that clinicians are often reluctant to discuss side effects when they perceive little can be done to attenuate these, especially when there are few or no therapeutic alternatives.

Third, some noted that identifying and quantifying the effect of side effects may be challenging. For instance, when a side effect is common, discussing the intensity and effect may be more meaningful to patients than simply describing the frequency or probability of occurrence. The PRO-CTCAE group noted that it was often important to adjust for baseline symptoms to maximize differences in patient-reported adverse events. The possibility of also using a single item to assess overall benefit-harm was discussed; a patient would be asked to rate whether the perceived benefit outweighed the effect on day-to-day life (i.e., was it worth it?).

Fourth, perceptions of benefit versus harm likely vary among subgroups and depending on individual circumstances. This theme is a current focus of the OMERACT Contextual Factors Working Group. For example, a person who is financially responsible for family members may be willing to tolerate more treatment-related symptoms if the medication allows them to continue working as compared with someone who does not have others relying on their ability to work. Inclusion of patients with diverse characteristics in race and ethnicity, age, sex, socioeconomic status, and living situations is needed in future trials to better understand issues related to safety priorities and tolerability.

There was also general agreement that in drug trials, competing priorities may influence the willingness of patients to disclose safety events. Some patients may be willing to tolerate more risk or be less likely to report adverse events so that they remain enrolled in trials that offer the only access to treatment, or if they perceive that the treatment is highly beneficial.

Personal non-commercial use only. The Journal of Rheumatology Copyright @ 2019 . All rights reserved. 


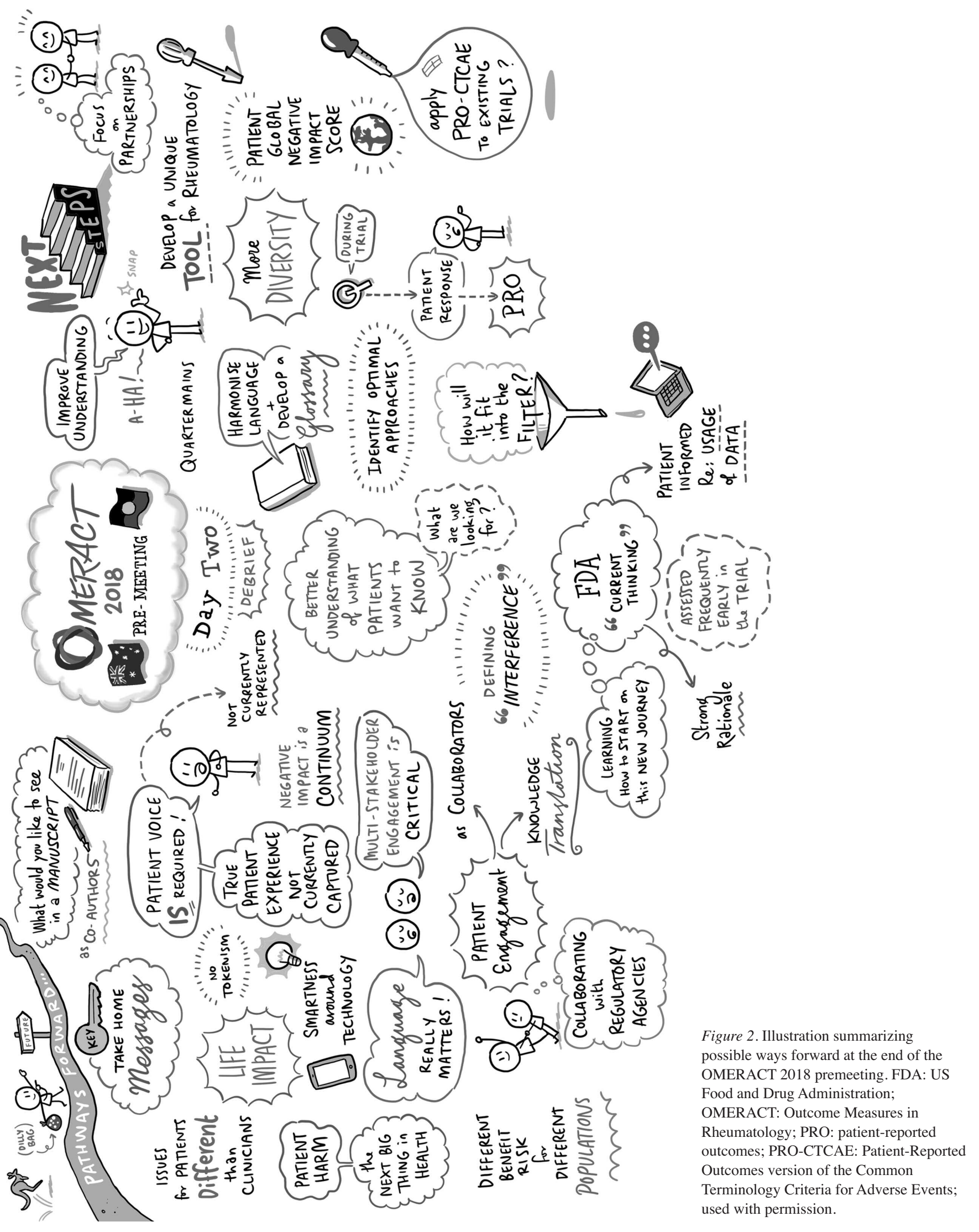

Personal non-commercial use only. The Journal of Rheumatology Copyright @ 2019. All rights reserved. 


\section{DISCUSSION}

Robust systems for designing, conducting, and reporting safety events in rheumatology RCT have been refined over many decades. However, to date, little attention has been given to understanding and measuring outcomes that matter most to patients. A better understanding is needed of how patients with rheumatic diseases view the relative benefits and potential harms of a treatment to design and select more adequate outcome measures for safety events monitoring. Such understanding can allow patients and clinicians to make informed choices about treatment and address longstanding challenges related to treatment initiation and longterm adherence. During the meeting, there was recognition that those involved view safety through various lenses. Indeed, the concept of safety seems inextricably linked to efficacy in that it is the relative balance of benefit and harm, rather than absolute frequency counts of symptoms, that may be most meaningful and informative to patients.

A research agenda to address this knowledge gap and develop patient-centered tools will require heightened appreciation for the full range of patient experiences, concerns, and preferences. The OMERACT Safety Group is currently conducting focus groups with international groups of patients to better elucidate patient perspectives and core domains needed to develop a new tool or adapt existing ones, such as the PRO-CTCAE. It will also be important to find ways to identify the cumulative negative effect of what have traditionally been viewed as "nuisance side effects," such as nausea, and address the added resources required to enhance collection, analysis, and interpretation of safety PRO. As with all OMERACT initiatives, it is essential that patients are fully engaged in the co-development and co-production of this work.

\section{ACKNOWLEDGMENT}

The authors acknowledge the contributions of Wen-Hung Chen, Tammy Clifford, Robert J. Holt, Peter Jones, Richard Veselý, and Andrew Wilson. The authors also acknowledge the contributions of the patient research partners.

\section{REFERENCES}

1. Boers M, Brooks P, Fries JF, Simon LS, Strand V, Tugwell P. A first step to assess harm and benefit in clinical trials in one scale. J Clin Epidemiol 2010;63:627-32.

2. Simon LS, Strand CV, Boers M, Brooks PM, Henry D, Tugwell PS. Observations from the OMERACT drug safety summit, May 2008. J Rheumatol 2009;36:2110-3.
3. Simon LS, Strand CV, Boers M, Brooks PM, Tugwell PS, Bombardier $\mathrm{C}$, et al. How to ascertain drug safety in the context of benefit. Controversies and concerns. J Rheumatol 2009;36:2114-21.

4. Lassere MN, Johnson KR, Boers M, Carlton K, Day RO, de Wit M, et al. Standardized assessment of adverse events in rheumatology clinical trials: Summary of the OMERACT 7 drug safety module update. J Rheumatol 2005;32:2037-41.

5. Boers M, Kirwan JR, Tugwell P, Beaton D, Bingham CO III, Conaghan PG, et al. The OMERACT Handbook. [Internet. Accessed September 20, 2018.] Available from: https://omeract.org/resources

6. Boers M, Kirwan JR, Wells G, Beaton D, Gossec L, d'Agostino MA, et al. Developing core outcome measurement sets for clinical trials: OMERACT filter 2.0. J Clin Epidemiol 2014;67:745-53.

7. Tong A, Craig JC, Nagler EV, Van Biesen W; SONG Executive Committee and the European Renal Best Practice Advisory Board; SONG Executive Committee and the European Renal Best Practice Advisory Board. Composing a new song for trials: the Standardized Outcomes in Nephrology (SONG) initiative. Nephrol Dial Transplant 2017;32:1963-6.

8. Kluetz PG, Chingos DT, Basch EM, Mitchell SA. Patient-reported outcomes in cancer clinical trials: measuring symptomatic adverse events with the national cancer institute's Patient-Reported Outcomes version of the Common Terminology Criteria for Adverse Events (PRO-CTCAE). Am Soc Clin Oncol Educ Book 2016; 35:67-73.

9. Standardised Outcomes in Nephrology. The SONG handbook [Internet. Accessed October 31, 2018.] Available from: http://songinitiative.org/reports-and-publications/

10. Dueck AC, Mendoza TR, Mitchell SA, Reeve BB, Castro KM, Rogak LJ, et al; National Cancer Institute PRO-CTCAE Study Group. Validity and reliability of the US national cancer institute's Patient-Reported Outcomes version of the Common Terminology Criteria for Adverse Events (PRO-CTCAE). JAMA Oncol 2015;1:1051-9.

11. Basch E, Reeve BB, Mitchell SA, Clauser SB, Minasian LM, Dueck AC, et al. Development of the national cancer institute's Patient-Reported Outcomes version of the Common Terminology Criteria for Adverse Events (PRO-CTCAE). J Natl Cancer Inst 2014;106.

12. Hay JL, Atkinson TM, Reeve BB, Mitchell SA, Mendoza TR, Willis G, et al; NCI PRO-CTCAE Study Group. Cognitive interviewing of the US National Cancer Institute's Patient-Reported Outcomes version of the Common Terminology Criteria for Adverse Events (PRO-CTCAE). Qual Life Res 2014;23:257-69.

13. Evangelidis N, Tong A, Manns B, Hemmelgarn B, Wheeler DC, Tugwell P, et al; Standardized Outcomes in NephrologyHemodialysis (SONG-HD) Initiative. Developing a set of core outcomes for trials in hemodialysis: an international Delphi survey. Am J Kidney Dis 2017;70:464-75.

14. Dueck AC, Mitchell SA, Rogak L, Ginos B, Sargent D, Shi Q, et al. A cluster-randomized study of clinician-patient shared vs standard reporting of symptomatic adverse events using PRO-CTCAE nested in a multicenter trial of multimodal therapy for rectal cancer (alliance n1048 PROSPECT). Qual Life Res 2015;24:1-2. 